ISSN 0103-8478

\title{
O crescimento da Nectandra megapotamica Mez., em floresta nativa na depressão central do Estado do Rio Grande do Sul
}

\author{
The growth of the Nectandra megapotamica Mez., in natural \\ forest in the central depression of the State of \\ the Rio Grande do Sul, Brazil
}

Helio Tonini ${ }^{1}$ Cesar Augusto Guimarães Finger ${ }^{2}$ Paulo Renato Schneider $^{3}$

\section{RESUMO}

Este trabalho foi realizado com o objetivo de estudar o crescimento em diâmetro, volume comercial, fator de forma comercial e incremento corrente anual do volume comercial em porcentagem (ICA\%) para duas árvores dominantes de canelapreta (Nectandra megapotamica), em uma Floresta Estacional Decidual, localizada no municipio de Santa Maria na depressão central do Estado do Rio Grande do Sul. Os dados foram obtidos mediante análise de tronco e as tendências de crescimento em diâmetro, volume comercial, fator de forma e ICA\%, foram ajustados com a utilização dos modelos de Mitscherlich \& Sontag (1982), Backman (1943), Richards (1959), e um modelo parabólico do segundo grau. O ajuste dos modelos indicou que, para as variáveis diâmetro e volume comercial, o melhor modelo foi o de Mitscherlich \& Sontag, para a árvore 1,sendo que, para a árvore 2, o modelo de Richards apresentou melhor ajuste. Para o incremento corrente anual do volume comercial em porcentagem, o melhor modelo foi o de Backmam, sendo o modelo parabólico, o de melhor ajuste para ofator de forma comercial.

Palavras-chave: Nectandra megapotâmica, análise de tronco, crescimento.

\section{ABSTRACT}

This work was carried out with the objective of studying the growth in diameter, commercial volume, form factor, mean annual increment of the commercial volume in percentage for two dominant trees of Nectandra megapotamica, in a Decidual Seasonal Forest, located in the municipal district of Santa Maria in the central depression of the State of Rio Grande do Sul, Brazil. The data were obtained from stem analysis and the growth trend in diameter, commercial volume, form factor and mean annual increment of the commercial volume in percentage, were fitted using the models of Mitscherlich \& Sontag (1982), Backman (1943), Richards (1959), and a second degree parabolic model. The adjustment of the models indicated that for the diameter and commercial volume, the best model was that one from Mitscherlich \& Sontag, for tree number one and for tree number two, the Richard's model presented better adjustment. For the mean annual increment of the commercial volume in percentage, the best model was that one from Backmam, being the parabolic model better for the form factor.

Key words: Nectandra megapotamica, stem analysis, growth.

\section{INTRODUÇÃO}

O consumo desordenado e a substituição de florestas por outras atividades de uso da terra levaram as reservas florestais nativas à exaustão, com uma rápida diminuição da cobertura florestal no Estado. A

\footnotetext{
${ }^{1}$ Engenheiro Florestal, doutorando pelo programa de Pós-graduação em Engenharia Florestal, Centro de Ciências Rurais, Universidade Federal de Santa Maria. E-mail: htonini@uol.com.br. Autor para correspondência.

${ }^{2}$ Engenheiro Florestal, Doutor, Professor Adjunto do Departamento de Ciências Florestais, Centro de Ciências Rurais, Universidade Federal de Santa Maria, 97105-900, Santa Maria, RS.

${ }^{3}$ Engenheiro Florestal, Doutor, Professor Titular do Departamento de Ciências Florestais, Centro de Ciências Rurais, Universidade Federal de Santa Maria, 97105-900, Santa Maria, RS.
} 
velocidade com que este importante recurso natural foi e vem sendo dilapidado, faz com que informações sobre a ecologia e o crescimento de nossas principais essências nativas sejam de fundamental importância para o reflorestamento e o manejo racional, de forma a se evitar os erros cometidos no passado.

Em um plano de manejo de florestas naturais, devem ser levados em consideração: a composição florística, a diversidade de espécies, a estrutura da floresta, o crescimento dos indivíduos, o recrutamento e a mortalidade, e todo o processo dinâmico de recomposição e reestruturação da floresta.

As pesquisas sobre o crescimento de espécies arbóreas individuais ou grupos de espécies nativas, sempre foram mais difíceis pela falta de anéis de crescimento visíveis. Segundo LAMPRECHT (1990), em árvores desprovidas de anéis de crescimento visíveis, a determinação do crescimento é ainda mais difícil e só se obtêm dados confiáveis com a realização de medições diretas periódicas por vários anos em parcelas permanentes.

A análise de tronco em árvores nativas com anéis visíveis é uma alternativa rápida e eficaz de se estudar as dimensões alcançadas pelas árvores em épocas passadas e obter informações importantes sobre parâmetros biométricos para descrever o desenvolvimento de uma determinada espécie, como o diâmetro, a altura e o volume.

Uma vez que o crescimento da canela-preta (Nectandra megapotamica)em relação às principais variáveis dendrométricas é desconhecido, este trabalho tem como objetivo mostrar a viabilidade de realização da análise de tronco para esta espécie e estudar o crescimento em diâmetro, volume comercial, fator de forma comercial e incremento corrente anual do volume comercial em porcentagem, permitindo uma avaliação do tempo necessário para que uma árvore atinja uma determinada dimensão, fornecendo subsídios básicos para o manejo florestal.

\section{Caracterização da espécie}

A Nectandra megapotamica pertence à família das Lauraceas, sendo vulgarmente conhecida como canela-louro, canela-preta, canela-ferrugem e canela-fedorenta. Amplamente dispersa pelas principais florestas do Estado, ocorre na Floresta do Alto Uruguai, nos sub-bosques dos pinhais do Planalto, na Floresta da Fralda da Serra Geral, na Floresta Pluvial Atlântica e no Escudo Sul-Rio-Grandense (REITZ et al.,1988).

Segundo LORENZI (1998), trata-se de uma planta perenifoliada ou semidecídua, sem preferência definitiva por tipo de solo, desenvolvendo-se em solos úmidos até os de drenagem rápida. Apresenta ampla dispersão pela floresta ombrófila em geral, sendo menos freqüente nas associações pioneiras e secundárias, e muito rara nos sub bosques dos pinhais e capões.

Para REITZ et al. (1983), pertence ao pequeno grupo arbóreo que se encontra em ótima vitalidade no interior da floresta densa e desenvolvida, onde ao lado das árvores adultas e velhas constata-se uma vigorosa regeneração junto com árvores medianamente desenvolvidas, demostrando se encontrar em pleno equilíbrio dinâmico. Por se tratar de espécie de sombra, sua instalação na vegetação secundária se inicia nos capoeirões.

A madeira é moderadamente pesada, fácil de trabalhar porém com cheiro desagradável quando fresca. Apresenta a superfície irregularmente lenhosa, de média durabilidade sob condições naturais (LORENZI, 1998).Apresenta nítida diferença entre cerne e alburno que é amarelo-ocráceo, sendo o cerne cor castanha, mais claro quando recém cortado (REITZ et al., 1983).

A madeira é adequada para a construção civil, esquadrias e tabuados em geral. Apesar das excelentes características xilotecnológicas, essa madeira tem sido relegada para segundo plano devido ao cheiro desagradável, que pode voltar quando em lugares úmidos (LORENZI, 1998).

Segundo REITZ et al. (1983), no entanto, já existem processos para neutralizar este mau cheiro e assim sendo, esta madeira como todas as canelas (Lauraceas) poderia proporcionar numerosas aplicações que requerem madeira suave, não muito pesada e resistente.

Trata-se de uma árvore muito ornamental, principalmente pela forma arredondada da copa, o que tem motivado seu largo uso na arborização de ruas de inúmeras cidades nos Estados de São Paulo e Paraná. Seus frutos são muito procurados por inúmeras espécies de pássaros, sendo ótima para reflorestamentos mistos de áreas de preservação permanente (LORENZI,1998).

\section{MATERIAL E MÉTODOS}

As árvores de canela-preta (Nectandra megapotamica) utilizadas neste estudo, originaramse de uma Floresta Estacional Decidual, localizada no Campo de Instrução de Santa Maria (CISM), na localidade do Boi Morto, no município de Santa Maria no Estado do Rio Grande do Sul.

A área em estudo pertence à Unidade de Mapeamento São Pedro e segundo BRASIL (1983), caracteriza-se por apresentar solo Podzólico Verme- 
lho Amarelo de textura média com substrato arenítico e argila de baixa atividade.

Segundo a classificação de Köppen, a região apresenta clima do tipo Cfa que é caracterizado por apresentar chuvas durante todos os meses do ano e possuir a temperatura média do mês mais quente superior a $22^{\circ} \mathrm{C}$ e a do mês mais frio superior a $3^{\circ} \mathrm{C}$.

No passado, este remanescente florestal sofreu pressão antrópica, quando foram retirados os indivíduos de maior valor econômico. No entanto, a partir de 1970, com sua aquisição pelo Exército, esta área passou a ser preservada.

Com o objetivo de estudar o crescimento em diâmetro, volume comercial sem casca (volume do fuste sem bifurcações), fator de forma comercial e incremento corrente anual do volume comercial em porcentagem, foram utilizadas duas árvores do estrato dominante, que foram cortadas e delas, retiradas fatias da base, Dap e a níveis superiores até a altura comercial definida pela ocorrência de bifurcações. Foram retiradas 4 fatias por árvore, sendo que na árvore 1 as posições foram 0,$4 ; 1,3 ; 5$ e 9,5 metros e na árvore $2,: 0,15 ; 1,3 ; 4,1$ e 7,6 metros.

Estas fatias foram secas em estufa apenas com a utilização de ventilação forçada. Posteriormente as mesmas foram lixadas com a utilização de uma lixa grossa (grão 60) e uma fina (grão 100).

Os anéis de crescimento foram considerados como anuais, devido às condições climáticas locais, que apresentam duas estações distintas, caracterizadas por um período de baixa atividade cambial no inverno ocasionando o surgimento de um lenho tardio visível.

A medição dos anéis de crescimento foi feita com o auxílio do aparelho LINTAB II, sobre 4 raios marcados em cada fatia, sendo os mesmos marcados em ângulos fixos de $90^{\circ}$. O volume comercial foi determinado pela cubagem anual das seções tomadas até a altura comercial entre as fatias através do método de Smalian, e o fator de forma comercial pela razão entre o volume comercial calculado e o volume cilíndrico tomando-se o Dap como referência.

Para estudar as tendências de crescimento em diâmetro, volume comercial, fator de forma e o incremento corrente anual em volume comercial expresso em porcentagem (ICA \%), foram utilizados quatro equações de regressão, apresentados na tabela 1 . Os ajustes foram feitos através do programa SPSS (Statistical Package for Social Science), sendo as equações selecionadas através da análise do coeficiente de determinação, erro padrão de estimativa percentual e análise de distribuição dos resíduos.

\section{RESULTADOS E DISCUSSÃO}

\section{Análise do crescimento}

Os resultados estatísticos do ajuste das equações testadas para estimar o crescimento em diâmetro e volume comercial em função da idade encontram-se apresentados na tabela 2 .

Para o crescimento em diâmetro e em volume, a análise do coeficiente de determinação, o coeficiente de variação e a distribuição dos resíduos, indicou que para a árvore 1,o modelo de Mitscherlich \& Sontag, apresentou melhor precisão estatística. Para a árvore 2, o modelo de Richards com três coeficientes, apresentou o melhor ajuste tanto para o crescimento em diâmetro, como para o crescimento em volume.

$\mathrm{Na}$ figura 1, estão representados as tendências de crescimento em diâmetro e volume comercial em função da idade, obtidos através do ajuste da função de Mitscherlich \& Sontag para a árvore 1 e da função de Richards com três coeficientes para a árvore 2 .

A análise desta figura mostra que, mesmo sendo árvores dominantes, com praticamente a mesma idade (48 e 52 anos) e não havendo diferença de sítio perceptível (as árvores foram coletadas a uma distância de aproximadamente 15 metros), existe uma nítida diferença no crescimento entre as duas árvores, sendo que a árvore 1 apresenta o crescimento em diâmetro e volume ainda em franca expansão, desenvolvendo-se de forma exponencial, ao contrário da árvore 2, que apresenta uma desaceleração no crescimento a partir dos 30 anos.

Com respeito a padrões de crescimento, TETTO \& SANQUETA (1996), ao estudarem a estrutura etária e padrões de crescimento em uma floresta 
Tabela 2: Parâmetros estatísticos das equações testadas para estimar o crescimento em diâmetro e volume comercial em função da idade.

\begin{tabular}{|c|c|c|c|c|c|c|c|c|c|c|}
\hline \multirow[b]{2}{*}{ Árvore } & \multirow[b]{2}{*}{ equação } & \multicolumn{9}{|c|}{ Diâmetro } \\
\hline & & $\mathrm{b}_{0}$ & $\mathrm{~b}_{1}$ & $\mathrm{~b}_{2}$ & $\mathrm{~b}_{3}$ & $\mathrm{~A}$ & $\mathrm{~K}$ & $\mathrm{R}$ & $\mathrm{R}^{2}$ & $\mathrm{CV} \%$ \\
\hline 1 & 1 & 0.1134 & -1.2444 & & 1.8137 & -- & -- & -- & 0.99 & 2.71 \\
\hline 1 & 2 & -1.4202 & 0.4946 & 0.2112 & -- & -- & -- & -- & 0.99 & 3.11 \\
\hline 1 & 3 & -0.3433 & 0.1206 & 0.0135 & -- & -- & -- & -- & 0.99 & 2.94 \\
\hline 2 & 1 & 0.3531 & 0.5639 & -- & 1.0413 & -- & -- & -- & 0.95 & 14.50 \\
\hline 2 & 2 & -1.0667 & 1.1273 & 0.0218 & -- & -- & -- & -- & 0.97 & 0.84 \\
\hline 2 & 3 & -4.3217 & 1.0718 & -0.0057 & -- & -- & -- & -- & 0.96 & 12.70 \\
\hline 2 & 4 & -- & -- & -- & -- & 35.726 & 0.0854 & 4.823 & 0.98 & 6.56 \\
\hline \multirow[b]{2}{*}{ Árvore } & & \multicolumn{9}{|c|}{ Volume comercial sem casca } \\
\hline & equação & $\mathrm{b}_{0}$ & $\mathrm{~b}_{1}$ & $\mathrm{~b}_{2}$ & $\mathrm{~b}_{3}$ & $\mathrm{~A}$ & $\mathrm{~K}$ & $\mathrm{R}$ & $\mathrm{r}^{2}$ & $\mathrm{CV} \%$ \\
\hline 1 & 1 & 0.3566 & -11.369 & -- & 3.1189 & -- & -- & -- & 0.99 & 3.69 \\
\hline 1 & 2 & -13.674 & 2.3930 & 0.3012 & -- & -- & -- & -- & 0.97 & -13.46 \\
\hline 1 & 3 & 0.0585 & -0.011 & 0.0005 & -- & -- & -- & -- & 0.99 & 4.89 \\
\hline 2 & 1 & 0.0012 & -0.0962 & -- & 1.6507 & -- & -- & -- & 0.95 & 19.25 \\
\hline 2 & 2 & -12.436 & 3.4392 & -0.0763 & -- & -- & -- & -- & 0.98 & -12.99 \\
\hline 2 & 3 & -0.0923 & 0.0083 & 0.0001 & -- & -- & -- & -- & 0.96 & 18.30 \\
\hline 2 & 4 & -- & -- & -- & -- & 0,631 & 0,111 & 16,217 & 0,98 & 7,2 \\
\hline
\end{tabular}

Onde: $b_{0}, b_{1}, b_{2}, b_{3}, A, K$ e $R=$ coeficientes; $R^{2}=$ coeficiente de determinação; $C V \%$ = coeficiente de variação.

natural com Araucaria angustifolia no estado do Paraná, verificaram a presença de 4 padrões básicos de crescimento nas árvores amostradas, determinadas em grande parte pelo seu posicionamento ao longo do perfil vertical da estratificação da floresta.

Sabidamente ocorrem variações de crescimento entre espécies, assim como podem haver variações dentro de uma mesma espécie, ou mesmo entre indivíduos, pois além da influência genética, provavelmente o tamanho e as condições de iluminação da copa e concorrência entre estes dois indivíduos foram diferentes ao longo de sua vida. Portanto a análise das dimensões e a concorrência em relação às árvores vizinhas provavelmente darão uma resposta para esta diferença na tendência de crescimento.

O crescimento das árvores, além do tempo, também depende das condições ambientais, que
ARVORE 1

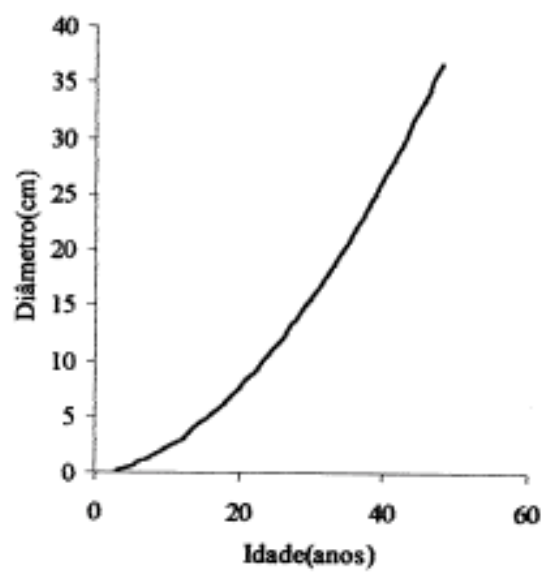

ÁRVORE 1

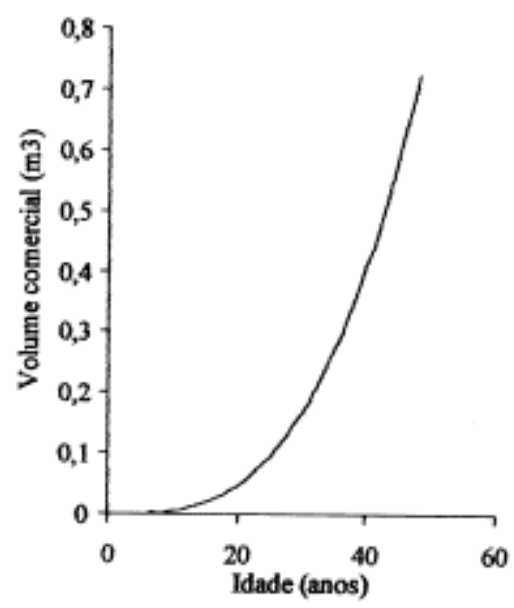

ÁRVORE 2

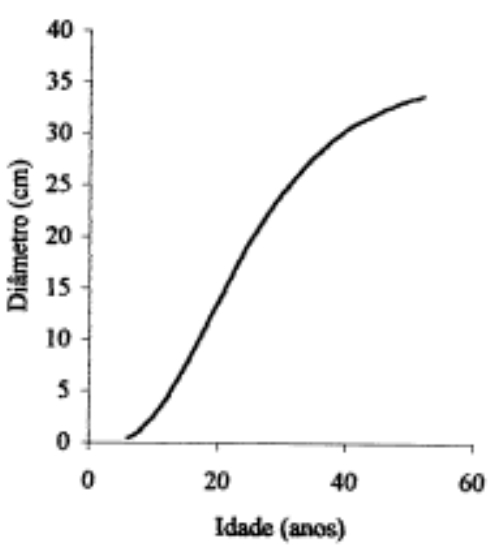

ÁRVORE 2

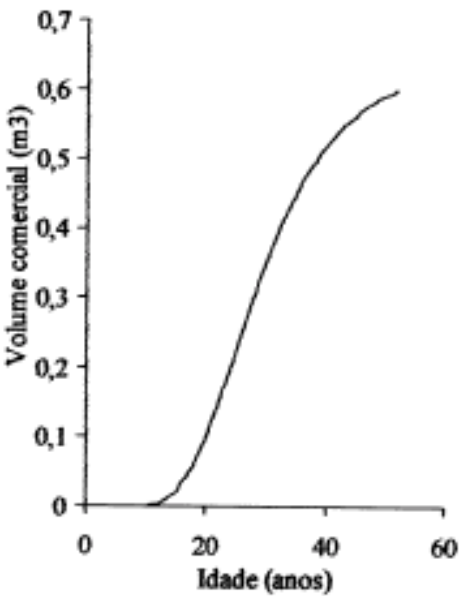

Figura 1 - Curva de crescimento em diâmetro e volume comercial para as árvores analisadas.

Ciência Rural, v. 33, n. 1, jan-fev, 2003. 
sendo favoráveis em um determinado período possibilitam o máximo rendimento. Em florestas nativas, a dinâmica da sucessão faz com que as condições ecológicas sejam diferenciadas para cada indivíduo ao longo do tempo.

As equações 1 a 3 da tabela 1 foram também ajustadas para descrever o ICA\% e o fator de forma comercial utilizando o dap como variável independente. Para o Incremento corrente anual do volume comercial em porcentagem, o modelo que apresentou melhor precisão estatística para as duas árvores analisadas foi o de Backman (2), com um coeficiente de determinação $\left(\mathrm{R}^{2}\right)$ de 0,86 e 0,89 e um $\mathrm{CV} \%$ de 1,34 e 2,09 para as árvores 1 e 2 respectivamente. Para o Fator de forma comercial, também para as duas árvores, o melhor modelo foi o de número três, com um coeficiente de determinação de 0,78 e 0,97 e CV\% de 4,4 e 2,77 para as árvores 1 e 2 respectivamente.

$\mathrm{Na}$ figura 2, que mostra o comportamento do ICA \%, observa-se que o incremento corrente anual do volume comercial em porcentagem para a árvore 1 , apresentou a forma exponencial negativa, diminuindo
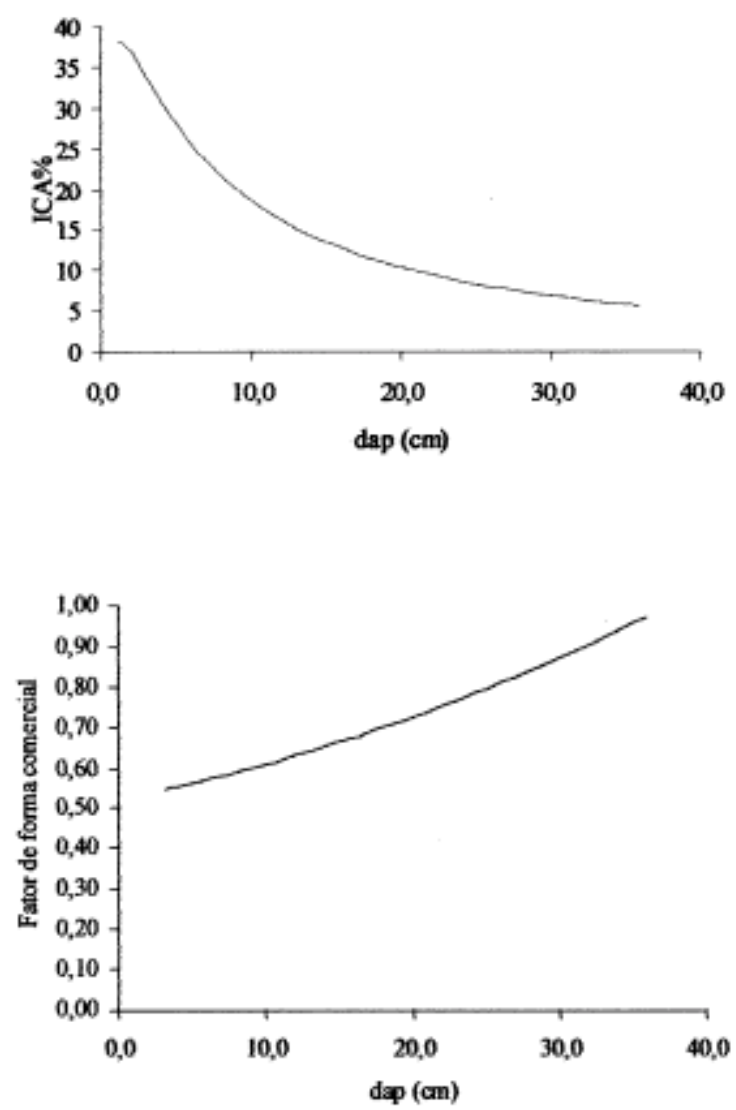

Figura 2 - Incremento corrente anual do volume comercial em porcentagem e fator de forma comercial do dap para as árvores 1 e 2 respectivemente. progressivamente com o aumento do diâmetro, seguindo uma das leis fundamentais do crescimento na qual a taxa relativa é sempre decrescente (Medawar apud. ZEIDE, 1993). Nesta figura, também pode ser observado o comportamento crescente do fator de forma comercial em relação ao dap para a árvore 2 .

Valores crescentes do fator de forma comercial com o dap, também foram encontrados por DURLO \& DENARDI (1998), para Cabralea canjerana, por SCHNEIDER et al. (2000) para Helietta apiculata, por SPATHELF et al.(no prelo), para árvores dominantes de Ilex brevicuspis, Erythoxilum deciduum, Cytharexylum montevidense e Rapanea ferruginea, e por SCHNEIDER et al. (2001), para Luehea divaricata.

Para a utilização prática, a tabela 3 mostra os valores médios para o diâmetro à altura do peito, volume comercial, ICA\% e fator de forma comercial.

Tabela 3: Valores médios para dap, volume comercial sem casca $\left(\mathrm{Vc}_{\mathrm{sc}}\right)$, incremento corrente anual do volume comercial em porcentagem (ICA\%) e fator de forma comercial $\left(\mathrm{f}_{\mathrm{c}}\right)$, para as duas árvores dominantes de Nectandra megapotamica analisadas.

\begin{tabular}{ccccc}
\hline Idade (anos) & dap $(\mathrm{cm})$ & $\mathrm{Vc}_{\mathrm{sc}}\left(\mathrm{m}^{3}\right)$ & $\mathrm{ICA} \%$ & $\mathrm{f}_{\mathrm{c}}$ \\
\hline 5 & 2,1 & 0,000289 & 50,8 & 0,36 \\
10 & 3,6 & 0,002287 & 45,7 & 0,41 \\
15 & 5,1 & 0,008094 & 35,4 & 0,42 \\
20 & 9,8 & 0,032406 & 30,8 & 0,69 \\
25 & 15,4 & 0,100571 & 17,5 & 0,79 \\
30 & 20,7 & 0,216132 & 10,7 & 0,80 \\
35 & 24,5 & 0,322934 & 5,0 & 0,82 \\
40 & 28,1 & 0,430022 & 6,7 & 0,83 \\
45 & 32,0 & 0,558529 & 4,7 & 0,88 \\
\hline
\end{tabular}

\section{CONCLUSÕES}

A partir dos resultados obtidos no presente trabalho conclui-se que:

a Nectandra megapotamica apresenta anéis de crescimento visíveis, que permitem a realização de análise de tronco;

as curvas de crescimento em diâmetro e volume comercial apresentaram diferenças para as duas árvores analisadas, sendo que a árvore 1 apresentou um crescimento ainda na fase exponencial e a árvore 2, já na forma sigmoidal típica;

o modelo de Mitscherlich \& Sontag apresentou o melhor ajuste para o diâmetro a altura do peito e volume comercial para árvore 1 . Para a árvore 2,0 melhor ajuste para as duas variáveis foi obtido com o modelo de Richards com três coeficientes;

o incremento corrente anual do volume comercial em porcentagem, apresentou tendência exponencial negativa para as duas árvores analisadas, 
mercial em porcentagem, apresentou tendência exponencial negativa para as duas árvores analisadas, sendo que o modelo de Backmam apresentou o melhor ajuste; para o fator de forma comercial, o melhor ajuste para as duas árvores em função do Dap, foi obtido com o modelo parabólico de segundo grau.

\section{REFERÊNCIAS BIBLIOGRÁFICAS}

BRASIL. Ministério da Agricultura. Inventário florestal nacional: Florestas nativas do Rio grande do Sul. Brasília, 1983. 345p.

INSTITUTO BRASILEIRO DE GEOGRAFIA E ESTATÍSTICA. Levantamento dos recursos naturais. Rio de Janeiro, 1986. 250p.

DURLO, M. A.; DENARDI, L. Morfometria de Cabralea canjerana em mata secundaria nativa do Rio Grande do Sul. Ciência Florestal, Santa Maria, v.8,n.1.p55-66,1998.

LAMPRECHT, H. Silvicultura nos trópicos: ecossistemas florestais e respectivas espécies arbóreas - possibilidades e métodos de aproveitamento sustentado. Dt. Ges. Für Techn. Zusammenarbeit (GTZ) Gmbh, Eschborn. [Tradução de Guilherme de Almeida Sedas e Gilberto Calcagnotto \}. Rossdorf: TZ- Verl.-Ges., 1990. 343p.

LORENZI, H. Árvores brasileiras: Manual de identificação e cultivo de plantas arbóreas nativas do Brasil. Nova Odessa : Plantarum, 1998. 368p.
REITZ, R; KLEIN, R. M; REIS, A. Projeto madeira do Rio Grande do Sul. Porto Alegre RS: Secretaria da Agricultura e de Abastecimento, 1983. 524p.

SCHNEIDER, P. S. P.; SCHEREN, L.W.; FLEIG, F. D. Crescimento da canela de veado Helietta apiculata Benth., na depressão central do Estado do Rio Grande do Sul. In: SIMPOSIO LATINOAMERICANO SOBRE MANEJO FLORESTAL, 1., 2000, Santa Maria. Anais... Santa Maria : UFSM, CCR, Programa de Pós-graduação em Engenharia Florestal, 2000. p.177189.

SCHNEIDER, P.S.P. et al. Crescimento do Açoita-cavalo (Luhea divaricata Mart.)., na depressão central do Estado do Rio Grande do Sul. In: SIMPOSIO LATINOAMERICANO SOBRE MANEJO FLORESTAL, 2., 2001, Santa Maria. Anais... Santa Maria : UFSM, CCR, Programa de Pós-graduação em Engenharia Florestal, 2001. p.487-507.

TETTO, A. F.; SANQUETTA, C. R. Estrutura etária e padrões de crescimento em uma floresta natural com Araucaria angustifolia (BERT.) O.KTZE., no Estado do Paraná. In: SIMPÓSIO INTERNACIONAL SOBRE ECOSSISTEMAS FLORESTAIS, 4., Belo Horizonte. Anais... Belo Horizonte : [s.n], 1996. p.227-228.

ZEIDE, B. Analysis of growth equations. Forest science, v.39,n.33. p.594-616, 1993. 